\title{
Alien hand sign in association with Alzheimer's histopathology
}

\author{
J A Ball, P L Lantos, M Jackson, C D Marsden, J W Scadding, M N Rossor
}

\begin{abstract}
A 68 year old man is described with an alien left hand, cortical myoclonus, bilateral parietal lobe dysfunction and memory impairment but preserved language skills. The clinical diagnosis was of corticobasal degeneration but at necropsy, four years after the onset of symptoms, the pathology was of Alzheimer's disease together with some scattered chromatolytic pale neurons in the cerebral cortex. The alien hand sign has not previously been described in Alzheimer's dementia and is an illustration of the clinical heterogeneity that may occur in association with Alzheimer histopathology.
\end{abstract}

$(\Im$ Neurol Neurosurg Psychiatry 1993;56:1020-1023)

Focal neurological signs are well recognised in Alzheimer's dementia and indeed Alzheimer's disease may present as a focal progressive cognitive disorder. Cases of necropsy proven Alzheimer's disease have been described presenting with progressive dysphasia, ${ }^{1}$ cortical blindness, ${ }^{2}$ a right parietal lobe syndrome ${ }^{3}$ and left hemiparesis. ${ }^{4}$ The "alien hand" sign, however, has not previously been described in association with Alzheimer pathology. This sign has been described in patients with callosal neoplasms and haemorrhage ${ }^{56}$ post callosotomy, ${ }^{7}$ in medial frontal cortex infarction, ${ }^{89}$ and in cases of trauma and haemorrhage affecting both the corpus callosum and medial frontal area. ${ }^{8-10}$ The syndrome has also been seen in corticobasal degeneration in which there may eventually be generalised cognitive impairment. ${ }^{11-15}$ We describe a patient presenting with bizarre uncooperative movements of the left arm characteristic of the "alien hand" syndrome, together with cortical myoclonus, bilateral parietal lobe dysfunction and global memory impairment but with relative preservation of speech. The clinical diagnosis was of corticobasal degeneration but necropsy revealed the features of Alzheimer's disease, together with some scattered chromatolytic pale neurons in the cerebral cortex.

\section{Case report}

A 68 year old right handed retired booking clerk presented with progressive difficulty in dressing. Two years before presentation he noticed difficulty tying his tie and later required his wife's help for all aspects of dressing. For nine months before presentation he had difficulty using a knife and fork and was using a fork alone. His writing had deteriorated and he was unable to put a key in the front door lock or negotiate a turnstile in the local supermarket. His wife had observed that he appeared to have particular difficulty using his left hand. She had noticed some deterioration in his memory but no speech impairment.

He had previously been very fit and was not on any medication. There was no family history of dementia or other neurological disease. His father died at the age of 36 years of renal failure. He had five siblings but no children. Blood pressure was $160 / 90$, cardiovascular, respiratory and abdominal examination were normal. On neurological examination there was a pout reflex and a left grasp reflex but no forced grasping or utilisation behaviour. There was myoclonus of both arms with multifocal distal muscle jerks present at rest and on voluntary movement. There was no startle, stimulus sensitive or reflex myoclonus. He had an incomplete left homonymous hemianopia. Voluntary saccades were impaired to the left, pursuit movements were full but saccadic. Optokinetic responses were reduced with the drum rotated to the right. The rest of the cranial nerves were normal. There was no cervical or axial rigidity. There was some dystonic posturing of the left hand together with bizarre uncooperative movements of the left arm particularly on attempting any task. The left hand would "wander" involuntarily, often making purposeless movements but did not show forced grasping or interfere with the actions of his right hand. These movements had the appearance of an "alien limb" to several examiners. When asked to perform a movement with the left arm there was either no response or an incorrect movement and the patient was aware that the movement was inappropriate. Tone was normal in the left arm. There was 
gegenhalten in the right hand but power and coordination of the right arm and leg were normal. Reflexes were symmetrical and normal and plantar responses were flexor. There was a left sensory inattention but no other abnormality on sensory testing. Gait was normal apart from the abnormal posture of the left arm.

Haematological and biochemical tests were normal including thyroid function tests and syphilis serology was negative. CSF examination was normal apart from an elevated protein at $1 \mathrm{~g} / \mathrm{L}$. Oligoclonal bands were negative. CT and MRI scans showed involutional changes only. Right carotid angiography was normal. EEG showed generalised theta activity. Small bowel biopsy showed no evidence of Whipple's disease.

Neuropsychological assessment was performed on four occasions. When first assessed on the Wechsler Adult Intelligence Scale (WAIS) ${ }^{16}$ verbal IQ was 91 and performance IQ 72. On the National Adult Reading Test $\left(\right.$ NART) ${ }^{17}$ reading IQ was 110 indicating that his WAIS scores were significantly below his optimum level. He scored only $2 / 24$ on a graded difficulty arithmetic test. There was mild impairment of both oral and written spelling (Baxter Graded Difficulty Spelling Test $10 / 30,<25$ th percentile). He had a marked apraxia bilaterally and was unable to learn a sequential hand movement. There was no orofacial apraxia. His visuospatial and perceptual skills were severely impaired affecting interpretation of complex visual material. He recognised only 9/20 Unusual Views and none of the 20 Fragmented Letters. He performed below the fifth percentile on visuospatial tests (Dot Centre 13/30, Dot counting 6/10). ${ }^{18} \mathrm{He}$ scored below the fifth percentile on the verbal version of the Recognition Memory Test and just above the chance level on the visual version. ${ }^{19} \mathrm{His}$ performance on tests of language was well preserved. Thus his vocabulary score on the WAIS was superior. He recognised 15/15 objects from their auditory descriptions. Assessment of nominal language skills was confounded by his perceptual difficulties. Nevertheless on the visually relatively easy Oldfield picture test, he named 19/26 objects. There was therefore evidence of focal cognitive deficits consistent with bilateral parietal lobe involvement and global memory impairment in the context of well preserved language skills.

When retested 7 months later his verbal IQ was 83 and his performance IQ only 58 . He was still able to read to an average level, but was now only able to spell easy words (3/30 on the Baxter test). His agraphia was now so severe that he was no longer able to write his name. His apraxia was such that he had difficulty using very common objects. He was profoundly acalculic, scoring only $0 / 24$ on the graded difficulty arithmetic test. His visuoperceptual skills had deteriorated to such a degree that he was quite unable to identify unusual views of objects. He was visually disorientated and unable to point accurately to dots in a random array. Assessed on easy shortened versions of the recognition memory tests for words and faces he only scored 16/25 and 14/25 respectively. In contrast his language functions remained well preserved WAIS vocabulary age scaled score 12 , Oldfield test $=16 / 30$, naming from description test $=12 / 15)$. Three months later little change was found. However, after a further eight months, 18 months since his initial assessment, there was evidence of further deterioration. His verbal IQ was now only borderline (76). His performance IQ could no longer be assessed. His visuo-perceptual and apraxic impairment had progressed to such a degree that it precluded formal assessment. He could no longer fixate on visually presented material nor was he able to point or perform other simple tests of praxis. Yet even at this advanced stage he was able to obtain an average score on the vocabulary subtest of the WAIS, indicating that his language skills continued to be well preserved.

The patient steadily deteriorated with worsening dyspraxia and confusion and aggressive behaviour but with relative preservation of speech. The features of the left alien hand became more exaggerated and the left hand became functionless. He required admission to hospital for long term care and died six months later of bronchopneumonia, two years eight months after presentation. The duration of symptoms of the disease was four years.

\section{NEUROPATHOLOGY}

The fixed weight of the brain was $1223 \mathrm{~g}$ and the brainstem and cerebellum weighed $155 \mathrm{~g}$. On external examination, the leptomeninges were thickened, particularly over the convexity. The large cerebral arteries showed a few, sometimes confluent atherosclerotic plaques and the lumina were narrowed up to $50 \%$. Cranial nerves were normal. Many sulci, particularly in the temporal lobes were widened. On coronal slices, the lateral ventricles and the third ventricle were moderately enlarged, and the angle of the lateral ventricles became rounded. The cerebral aqueduct was of normal size and shape. The Sylvian fissures were considerably enlarged and there was additional space between the hippocampus and the wall of the temporal horn of the lateral ventricles. The sulci were widened and the gyri narrowed throughout the cerebral hemispheres, most obviously in the temporal lobes. The cortical ribbon appeared narrowed. The substantia nigra was well pigmented, but the locus coeruleus was pale.

Blocks of tissue were taken from the frontal, temporal, parietal and occipital lobes, the corpus striatum, lentiform nucleus with the nucleus basalis of Meynert, thalamus with the subthalamic nucleus and lateral geniculate body, from the red nucleus, cerebellar vermis and hemisphere, midbrain, pons and medulla oblongata. Additional five blocks were taken from the premotor cortex and supplementary motor area. Altogether 21 samples of tissue were 


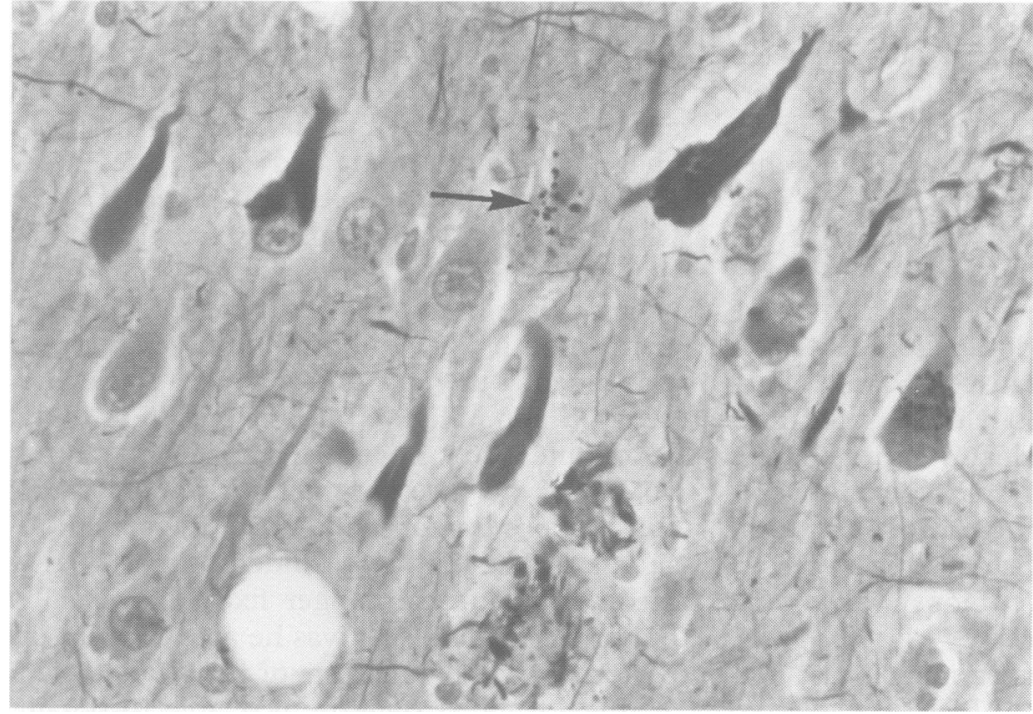

Figure Neurofibrillary tangles, neuritic plaques and granulo-vacuoles (arrow) in the hippocampus. Marsland-Glees silver impregnation. $\times 625$

examined. Paraffin-wax embedded sections were stained with haematoxylin and eosin, luxol-fast blue and cresyl violet, and Congo red, and impregnated with silver according to Marsland and Glees (fig). Immunocytochemistry was performed, using the avidinbiotin complex (Amersham) for glial fibrillary acidic protein (GFAP, DAKO), $\beta A 4$ protein (12-28), 147 neurofibrillary protein (both kindly provided by Professor BH Anderton, Institute of Psychiatry, London) and ubiquitin (DAKO). Histology showed many neurofibrillary tangles, senile plaques, granulovacuoles and a few Hirano bodies in the hippocampus. Plaques and tangles were also common throughout the neocortex, and tangles also occurred in the deep grey matter, including the nucleus basalis of Meynert, lentiform nucleus, thalamus, subthalamic nucleus, mamillary bodies and in the brainstem. Immunocytochemistry for $\beta$ A4 protein demonstrated abundant deposits in the neocortex, in excess of plaque formation as demonstrated by silver impregnation. There were a few, large, pale chromatolytic nerve cells in the neocortex, some with a preserved peripheral rim of Nissl substance. Many plaques and tangles, with prominent astrocytosis and a tiny old infarct were seen in the medial frontal cortex. The substantia nigra showed little loose pigment and an occasional tangle, but no neuronal loss or Lewy bodies. The neurons of the locus coeruleus were depleted and several tangles as well as an occasional pale cell were noted. There was neuronal loss in the nucleus basalis of Meynert. Patchy pallor was obvious on myelin stain in the white matter, particularly of the temporal and occipital lobes. In these areas and in stretches of the superficial cortex astrocytes were prominent; more obviously with GFAP immuno-stain. The blood vessels had somewhat thickened, fibrous walls, and several small and medium-sized leptomeningeal and parenchymal vessels contained amyloid. There were a few perivascular mono- nuclear cells, including pigment-containing macrophages.

\section{Discussion}

The striking progressive clinical features in this case were an alien left hand, dystonic posturing of the left hand, myoclonus, apraxia and bilateral parietal lobe dysfunction with impaired memory but relative preservation of speech. Despite some atypical features, the clinical diagnosis was thought to be that of corticobasal degeneration in view of the asymmetric motor features, the alien behaviour of the left hand, the dystonic posturing of that limb, the bilateral apraxia, and hemisensory disturbance. The degree of cognitive impairment to begin with was not dramatic, although the severe impairment of visuo-spatial and perceptual skills, as well as the hemianopia, were unusual. Nevertheless, corticobasal degeneration was considered most likely to be the diagnosis. However at necropsy the brain showed predominantly Alzheimer type pathology: senile plaques and neurofibrillary tangles were numerous both in the hippocampus and neocortex, and tangles occurred also in the deep grey matter and brainstem. There were a few, pale neurons in the cortex, but the overall appearances did not support the diagnosis of cortico-basal degeneration. ${ }^{11}$ Large, chromatolytic, socalled ballooned or swollen neurons have, however, been described previously in Alzheimer's disease, in addition to other neurodegenerative disorders. ${ }^{20}$ The case also had some vascular pathology with atherosclerosis of the large cerebral vessels and amyloid angiopathy as demonstrated by both Congo red and $\beta \mathrm{A} 4$ protein immunocytochemistry.

It is well recognised that Alzheimer's disease may present with focal neurological signs and the characteristic neuropathological findings may be confined to circumscribed areas of the brain. Language disorder, however, usually becomes prominent as the disease progresses whereas in this case speech was well preserved. As in this case myoclonus with the neurophysiological characteristics of cortical myoclonus occurs in Alzheimer's disease, but may occur in other forms of cortical dementia. However the "alien hand" sign has not previously been reported in Alzheimer's disease and focal motor symptomotology is uncommon and may lead to this diagnosis being considered unlikely.

We thank Mr A Chadwick of the MRC Alzheimer's Disease Brain Bank for skilful technical assistance. This work was supported by the MRC.

1 Pogacar S, Williams RS. Alzheimer's disease presenting as slowly progressive aphasia. Rhode Is Med $\mathcal{F}$ 1984;67: 181-5.

2 Faden AI, Townsend J. Myoclonus in Alzheimer Disease A confusing sign. Arch Neurol 1976;33:278-80.

3 Crystal HA, Horoupian DS, Katzman R, Jotkowitz S. Biopsy-proven Alzheimer disease presenting as a right parietal lobe syndrome. Ann Neurol 1982;12:186-8.

4 Jagust WJ, Davies P, Tiller-Borcich JK, Reed BR. Focal Alzheimer's disease. Neurology 1990;40:14-9.

5 Brion S, Jedynak CP. Trouble du transfert interhemispherique a propos de trois observations de tumeurs du corps calleux; Le signe de la main etrangere. Rev Neurol (Paris) 1972;126:257-66. 
6 Leiguarda R, Starkstein S, Berthier M. Anterior collosal haemorrhage. A partial interhemispheric disconnection haemorrhage. A partial interhemisphe

7 Wilson DH, Reeves A, Gazzaniga M. Cerebral commisurotomy for control of intractable seizures. Neurology 1977;27:708-15.

$8 \mathrm{McNabb}$ AW, Carroll WM, Mastalgia FL. "Alien" hand and loss of bimanual coordination after dominant anterior cerebral artery territory infarction. $\mathcal{f}$ Neurol Neurosurg Psychiatry 1988;51:21 8-22.

9 Mukai T, Higasitani N, Hashimoto A, Hitomi $\mathrm{K}$, Hanada M. A case of alien hand. Clin Neurol 1988;28. 869-73.

10 Banks G, Short P, Martinez J, Latchaw R, Ratcliff G, Boller $\mathrm{F}$. The alien hand syndrome clinical and postmortem findings. Arch Neurol 1989;46:456-60.

11 Gibb WRG, Luthert PJ, Marsden CD. Corticobasal degeneration. Brain 1989;112:1171-93.

12 Rebeiz J, Kolodny EH, Richardson Jr EP. Corticodentatonigral degeneration with neuronal achromasia. Arch Neurol 1968;18:20-33.

13 Riley DE, Lang AE, Resch L, Ashby P; Hornyckiewicz O,
Black S. Cortico-basal ganglionic degeneration. Neurology 1990;40:1203-13.

14 Sawle GV, Brooks DJ, Marsden CD, Frackowiak RSJ. Corticobasal degeneration. A unique pattern of regional cortical oxygen hypometabolism and striatal fluorodopa uptake demonstrated by positron emission tomography. Brain 1991;114:541-57.

15 Scully $R$. Case records of the Massachusetts General Hospital (Case 38). N Engl fु of Med 1985;313:739-48.

16 Wechsler D. Wechsler Adult Intelligence Scale. New York: The Psychological Corporation, 1955.

17 Nelson HE. The National Adult Reading Test. Windsor, UK: NFER-Nelson, 1982.

18 Warrington EK, James M. Visual apperceptive agnosia: a clinico-anatomical study of three cases. Cortex 1988; 24:13-32.

19 Warrington EK. The recognition memory test manual. Windsor, UK: NFER-Nelson, 1984

20 Dickson DW, Yen S-H, Suzuki KI, Davies P, Garcia JH, Hirano A. Ballooned neurons in select neurodegenerative diseases contain phosphorylated neurofilament epitopes. Acta Neuropathol (Berl) 1986;71:216-23. 